\title{
Dust-Acoustic Solitary Waves in a Magnetized Dusty Plasma with Ions of Distinct Temperatures
}

\author{
M. G. M. Anowar ${ }^{1}$, I. Tasnim ${ }^{1}$, and M. M. Masud ${ }^{2}$ \\ ${ }^{1}$ Department of Physics, Begum Rokeya University, Rangpur, Bangladesh. \\ ${ }^{2}$ Department of Physics, Bangladesh University of Engineering and Technology, Dhaka, Bangladesh.
}

\begin{abstract}
A theoretical investigation has been made of obliquely propagating electrostatic solitary structures in a magnetized dusty plasma, composed of negatively charged dust, nonthermal ions of distinct temperatures, and Boltzmann distributed electrons. The properties of small but finite amplitude dust-acoustic solitary waves (DASWs) in this dusty plasma system are studied by deriving the Zakharov-Kuznetsov (ZK) equation. It has been inferred that the effects of obliqueness, external magnetic field, nonthermal ions of two distinct temperatures, and Boltzmann electrons cause modification of the characteristics (i.e., amplitude and width) of these solitary structures. The implications of our present investigation in understanding the basic features of nonlinear electrostatic perturbations observed in many space plasma systems and laboratory devices are briefly discussed.
\end{abstract}

\section{Introduction}

Nowadays, the study of waves and their instabilities in dusty plasmas have been the focus of intense interest because of its vital role in understanding various types of collective processes in space and laboratory plasmas, such as cometary tails, planetary rings, intergalactic and interstellar media, Earth's environment, etc. [1-4]. The presence of charged dust grains in a plasma modifies the existing plasma wave spectra as well as introduces a number of novel eigenmodes [viz., dust-ion acoustic waves (DIAWs) [5, 6], dust-acoustic waves (DAWs) [7-10], dust lattice waves [11],etc.]. Dust grains acquire negative charge by the collection of electrons $[1,12]$ because the thermal speed of the electron is much higher than that of the ions. The phase speed of DAWs is much smaller than the electron and ion thermal speed but larger than the dust thermal speed in which restoring force is provided by the thermal pressure of ions and electrons while inertia is provided by the mass of dust. Recently, several authors [13-15] have analyzed the effects of negatively or positively charged dust grains on electrostatic waves in nonthermal dusty plasmas.

Generally, nonthermal plasma is a plasma which is not in thermodynamic equilibrium, either because the ion temperature is different from the electron temperature, or because the velocity distribution of one of the species does not follow a Maxwell-Boltzmann distribution. A number of spacecraft or satellite observations indicate the existence of nonthermal electrons or ions in different space plasma environments particularly, in/around the Earth's bow shock [16]and foreshock [17], in the upper Martainionsphere[18], in the auroral region $[19,20]$, in the lower part of magnetosphere $[21,22]$ etc. To explain the effects of nonthermal plasma species on the basic characteristics of the plasma system, containing fast nonthermal plasma species, Cairns et al. [23]introduced a distribution function followed by those plasma species. After this pioneering work of Cairns et al. a number of authors have studied the effects of nonthermal plasma species on different electrostatic propagation modes in a dusty plasma system containing negatively charged dust [5, 24, 25, 27]. The above cited works $[5,24,25,27]$ are confined to negatively charged dust and nonthermal ions or electrons of single temperature only. But the existence of two-temperature ions [26, 28, 29, 31-33, 41] or electrons [34, 36-38] which are common to occur in both space environments [36, 39, 40]and laboratory plasmas $[35,36]$ are the topic of recent interest. Recently, Tasnimet al. have studied the effects of distinct temperature nonthermal ions on DA solitary [26]and shock [41]structures, respectively, in unmagnetized dusty plasmas with negatively charged dust. Dorranian and Sabetkar[42] have also studied the properties of DA solitons considering an unmagnetized dusty plasma system containing negatively charged dust and two nonthermal ion species at different temperatures deriving the Kadomtsev-Petviashivili (KP) equation. The effects of external magnetic field, obliqueness, and arbitrarily charged dust on the solitary structures in dusty plasmas with ions of different temperatures are not discussed at all in these works [26, 41, 42]. In this investigation, we have considered the propagation of highly nonlinear dust-acoustic solitary waves (DASWs) in a magnetized dusty plasma consisting of negatively charged dust fluid, Boltzmann distributed electrons, and nonthermal ions of distinct temperatures. We have derived the Zakharov-Kuznetsov (ZK) equation [32, 43]for the considered dusty plasma model, and analyzed the properties of DASWs both analytically and numerically. The manuscript is organized in the following manner. In section II, the relevant equations governing the dynamics of nonlinear DAWs are 
provided. The ZK equation is derived in section III. The numerical solution of ZK equation is analyzed in section IV. A brief discussion of the results obtained from this investigation is contained in section V.

\section{Governing Equations}

We consider the nonlinear propagation of DA waves in a collisionless magnetized dusty plasma system consisting of negatively charged mobile dust fluid, nonthermal ions of distinct temperatures $T_{i l}$ and $T_{i 2}$, and noninertial electrons having finite temperature $T_{e}$, where $T_{e} \gg T_{i 2} \gg T_{i 1}$ in the presence of an external magnetic field $\mathbf{B}_{\mathbf{0}}=B_{0} \hat{z}$. Thus, at equilibrium, $n_{i 10}+n_{i 20}=n_{e 0}+Z_{d} n_{d 0}$, where $n_{i 10}$ and $n_{i 20}$ are the densities of the lower and higher temperature ions, respectively, at equilibrium, $Z_{d}$ is the number of electrons residing onto the dust grain surface, and $n_{e 0}\left(n_{d 0}\right)$ is the equilibrium density of the electron (dust). The nonlinear dynamics of the obliquely propagating DA waves in such a dusty plasma system is governed by

$$
\begin{aligned}
& \frac{\partial n_{d}}{\partial t}+\nabla \cdot\left(n_{d} \boldsymbol{u}_{\boldsymbol{d}}\right)=0, \\
& \frac{\partial \boldsymbol{u}_{\boldsymbol{d}}}{\partial t}+\left(\boldsymbol{u}_{\boldsymbol{d}} \cdot \boldsymbol{\nabla}\right) \boldsymbol{u}_{\boldsymbol{d}}=\boldsymbol{\nabla} \phi-\alpha\left(\boldsymbol{u}_{\boldsymbol{d}} \times \hat{z}\right)=0, \\
& \nabla^{2} \phi=n_{d}+\mu e^{\sigma_{2} \phi}-\mu_{i 1}\left(1+\beta \phi+\beta \phi^{2}\right) e^{-\phi}-\mu_{i 2}\left(1+\beta \sigma_{1} \phi+\beta \sigma_{1}^{2} \phi^{2}\right) e^{-\sigma_{1} \phi},(3)
\end{aligned}
$$

where $n_{d}$ is the dust particle number density normalized by its equilibrium value $n_{d 0}, \boldsymbol{u}_{d}$ is the dust fluid velocity normalized by $C_{d}=\left(Z_{d} T_{i l} / m_{d}\right)^{1 / 2}, \phi$ is the wavepotential normalized by $T_{i l} / e$, the time variablet is normalized by dust plasma period $\omega_{p d}{ }^{-1}=\left(m_{d} / 4 \pi n_{d 0} Z_{d}^{2} e^{2}\right)^{1 / 2}$, and the space variable is normalized by $\lambda_{D m}=\left(T_{i l} / 4 \pi n_{d 0} Z_{d} e^{2}\right)^{1 / 2}$. Here, $\sigma_{I}=T_{i l} / T_{i 2}, \sigma_{2}=T_{i l} / T_{e}, \alpha=\omega_{c d} / \omega_{p d}$ is the dust cyclotron frequency normalized by $\omega_{p d}, \beta$ is the nonthermal parameter, $\mu_{i l}=n_{i l} \delta Z_{d} n_{d 0}, \mu_{i 2}=n_{i 2} \delta Z_{d} n_{d 0}, \mu=n_{e} / Z_{d} n_{d 0}=\mu_{i 1}+\mu_{i 2}-1$, and $e$ is the magnitude of the electron charge.

\section{Derivation Of ZK Equation}

To study small but finite amplitude electrostatic DASWs in the dusty plasma system, under consideration, one usually constructs a weakly nonlinear theory [44], and uses a scaling of the independent variables through the stretched coordinates $[32,43,44]$ as

$X=\epsilon^{1 / 2} x$,

$Y=\epsilon^{1 / 2} y$,

$Z=\epsilon^{1 / 2}\left(z-V_{p} t\right)$,

$\tau=\epsilon^{3 / 2} t$

where $\epsilon$ is a small parameter measuring the weakness of the dispersion, $V_{p}$ is the phase speed normalized by the dust-acoustic speed $C_{d}$. It may be noted here that $X, Y$ and $Z$ are all normalized by the Debye radius $\left(\lambda_{D m}\right)$, and $\tau$ is normalized by the ion dust plasma period $\left(\omega_{p d}{ }^{-1}\right)$.

The perturbed quantities $n_{d}, u_{d x}, u_{d y}, u_{d z}$, and $\phi$ can be expanded along with their equilibrium values as [44-47]

$n_{d}=1+\epsilon n_{d}^{(1)}+\epsilon^{2} n_{d}^{(2)}+\cdots$,

$u_{d x}=\epsilon^{3 / 2} u_{d x}^{(1)}+\epsilon^{2} u_{d x}^{(2)}+\cdots$,

$u_{d y}=\epsilon^{3 / 2} u_{d y}^{(1)}+\epsilon^{2} u_{d y}^{(2)}+\cdots$,

$u_{d z}=\epsilon u_{d z}^{(1)}+\epsilon^{2} u_{d z}^{(2)}+\cdots$,

$\phi=\epsilon \phi^{(1)}+\epsilon^{2} \phi^{(2)}+\cdots$.

Now, using eqns. (4)-(7), and (8)-(12) into eqns. (1)-(3), one can obtain the first order continuity equation, $z$-component of the momentum equation, and Poisson's equation, which after simplification, yield $n_{d}^{(1)}=\frac{1}{V_{p}^{2}} \phi^{(1)}$,

$u_{d z}^{(1)}=\frac{1}{V_{p}} \phi^{(1)}$,

$V_{p}=\left[\frac{1}{\sqrt{\mu \sigma_{2}+\mu_{i 1}-\mu_{i 1} \beta+\mu_{i 2} \sigma_{1}-\mu_{i 2} \beta \sigma_{1}}}\right]$.

Eqn. (15) is the phase speed of theDA waves propagating in the magnetized dusty plasma, under consideration.

The first order $x$ - and $y$-components of the momentum equation can be written as

$u_{d x}^{(1)}=-\frac{1}{\alpha} \frac{\partial \phi^{(1)}}{\partial Y}$,

$u_{d y}^{(1)}=\frac{1}{\alpha} \frac{\partial \phi^{(1)}}{\partial X}$.

The equations (16) and (17), respectively, represent the $x$ and $y$-components of $\left(\mathbf{V}_{\mathrm{E}}+\mathbf{V}_{\mathrm{D}}\right)$ for dust, where $\mathbf{V}_{\mathrm{E}}$ and $\mathbf{V}_{\mathrm{D}}$ are $\mathbf{E} \times \mathbf{B}_{0}$ and diamagnetic drift, respectively. These equations are also satisfied by the second order continuity equation. 
Again, using eqns. (4)-(7) and (8)-(12) into eqns. (1)-(3), and eliminating $u_{d x, y}^{(1)}$, the next higher order $x$ and $y$-components of the momentumequation, and Poisson's equation can be found as

$u_{d x}^{(2)}=-\frac{V_{p}}{\alpha^{2}} \frac{\partial^{2} \phi^{(1)}}{\partial Z \partial X}$,

$u_{d y}^{(2)}=-\frac{V_{p}}{\alpha^{2}} \frac{\partial^{2} \phi^{(1)}}{\partial Z \partial Y}$

$$
\begin{aligned}
& \frac{\partial^{2} \phi^{(1)}}{\partial X^{2}}+\frac{\partial^{2} \phi^{(1)}}{\partial Y^{2}}+\frac{\partial^{2} \phi^{(1)}}{\partial Z^{2}}=n_{d}^{(2)}+\mu \sigma_{2} \phi^{(2)}+\frac{1}{2} \mu \sigma_{2}^{2}\left(\phi^{(1)}\right)^{2}-\mu_{i 1} \beta \phi^{(2)}+\mu_{i 1} \phi^{(2)} \\
&-\frac{1}{2} \mu_{i 1}\left(\phi^{(1)}\right)^{2}-\mu_{i 2} \beta \sigma_{1} \phi^{(2)}+\mu_{i 2} \sigma_{1} \phi^{(2)}-\frac{1}{2} \mu_{i 2} \sigma_{1}^{2}\left(\phi^{(1)}\right)^{2} .
\end{aligned}
$$

Equations (18) and (19), respectively, denote the $x$ - and $y$-components of the dust polarization drifts. Now, following the same procedure one can obtain the next higher order continuity equation, and $z$-component of the momentum equation. Using these new higher order equations along with (13)-(20), one can eliminate $n_{d}^{(2)}, u_{d z}^{(2)}$, and $\phi^{(2)}$, and can finally obtain

$\frac{\partial \phi^{(1)}}{\partial \tau}+A B \phi^{(1)} \frac{\partial \phi^{(1)}}{\partial Z}+\frac{1}{2} A \frac{\partial}{\partial Z}\left[\frac{\partial^{2}}{\partial Z^{2}}+D\left(\frac{\partial^{2}}{\partial X^{2}}+\frac{\partial^{2}}{\partial Y^{2}}\right)\right] \phi^{(1)}=0$,

where

$A=V_{p}^{3}$,

$B=\frac{1}{2}\left(\mu_{i 1}+\mu_{i 2} \sigma_{1}^{2}-\mu \sigma_{2}^{2}-\frac{3}{V_{p}^{4}}\right)$,

$D=1+\frac{1}{\alpha^{2}}$.

\section{SW Solution Of The ZK Equation}

To study the properties of the SWs propagating in a direction making an angle $\delta$ with the $Z$-axis, i.e., with the external magnetic field and lying in the $(Z-X)$ plane, the coordinate axes $(X, Z)$ are rotated through an angle $\delta$, keeping the $Y$-axis fixed. Thus, we transform our independent variables to

$\rho=X \cos \delta-Z \sin \delta$

$\eta=Y$

$\xi=X \sin \delta+Z \cos \delta$,

$\tau=t$.

This transformation of these independent variables allows us towrite the ZK equation in the form

$$
\begin{aligned}
\frac{\partial \phi^{(1)}}{\partial t}+\delta_{1} \phi^{(1)} \frac{\partial \phi^{(1)}}{\partial \xi}+\delta_{2} \frac{\partial^{3} \phi^{(1)}}{\partial \xi^{3}}+\delta_{3} \phi^{(1)} \frac{\partial \phi^{(1)}}{\partial \rho}+ & \delta_{4} \frac{\partial^{3} \phi^{(1)}}{\partial \rho^{3}}+\delta_{5} \frac{\partial^{3} \phi^{(1)}}{\partial \xi^{2} \partial \rho} \\
& +\delta_{6} \frac{\partial^{3} \phi^{(1)}}{\partial \xi \partial \rho^{2}}+\delta_{7} \frac{\partial^{3} \phi^{(1)}}{\partial \xi \partial \eta^{2}}+\delta_{8} \frac{\partial^{3} \phi^{(1)}}{\partial \rho \partial \eta^{2}}=0,
\end{aligned}
$$

where

$\delta_{1}=A B \cos \delta$,

$\delta_{2}=\frac{1}{2} A\left(\cos ^{3} \delta+D \sin ^{2} \delta \cos \delta\right)$,

$\delta_{3}=-A B \sin \delta$,

$\delta_{4}=-\frac{1}{2} A\left(\sin ^{3} \delta+D \sin \delta \cos ^{2} \delta\right)$,

$\delta_{5}=A\left[D\left(\sin \delta \cos ^{2} \delta-\frac{1}{2} \sin ^{3} \delta\right)-\frac{3}{2} \sin \delta \cos ^{2} \delta\right]$,

$\delta_{6}=-A\left[D\left(\sin ^{2} \delta \cos \delta-\frac{1}{2} \cos ^{3} \delta\right)-\frac{3}{2} \sin ^{2} \delta \cos \delta\right]$,

$\delta_{7}=\frac{1}{2} A D \cos \delta$,

$\delta_{8}=-\frac{1}{2} A D \sin \delta$.

It is now necessary to look for a steady state solution of this ZK equation in the form

$\phi^{(1)}=\phi_{0}(Z)$,

where

$Z=\xi-u_{0} t$

in which $u_{0}$ is a constant speed normalized by the positive DA speed $C_{d}$. Using this transformation the ZK equation can be written in steady state form as

$-u_{0} \frac{d \phi_{0}}{d Z}+\delta_{1} \phi_{0} \frac{d \phi_{0}}{d Z}+\delta_{2} \frac{d^{3} \phi_{0}}{d Z^{3}}=0$. 
Now, using the appropriate boundary conditions, viz., $\phi^{(l)} \rightarrow 0,\left(d \phi^{(1)} / d Z\right) \rightarrow 0,\left(d^{2} \phi^{(1)} / d Z^{2}\right) \rightarrow 0$ as $Z \rightarrow \pm \infty$, the solitary wave solution of this equation is given by

$\phi_{0}(Z)=-\phi_{m} \operatorname{sech}^{2}(\kappa Z)$,

where $\phi_{m}=3 u_{0} / \delta_{1}$ is the magnitude of amplitude and $\kappa=\sqrt{u_{0} / 4 \delta_{2}}$ is the inverse of the width of the solitary waves. As $A>0$, it is clear from eqns.(23) and (27) that depending on whether $B$ is positive or negative, the SWs will be associated with either positive potential $\left(\phi_{m}>0\right)$ or negative potential $\left(\phi_{m}<0\right)$. Therefore, there exist SWs associated with positive (negative) potential when $B>0(B<0)$.

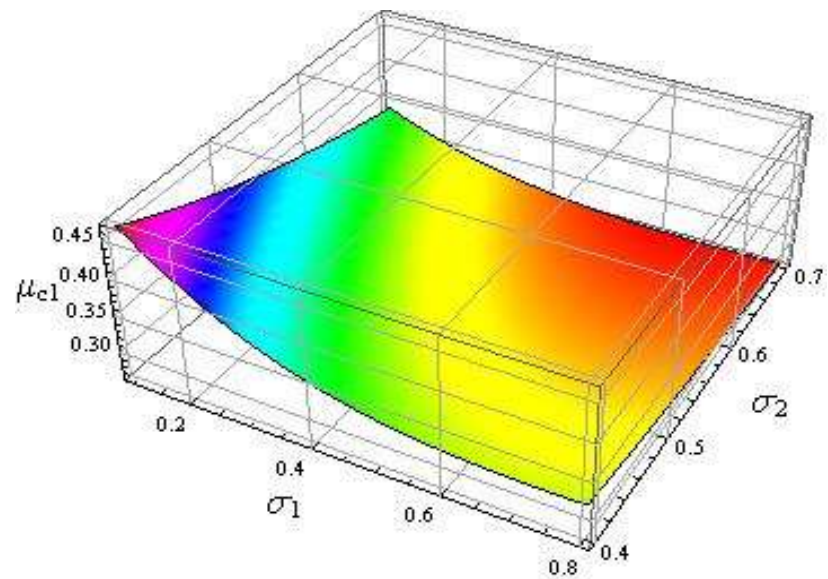

Figure 1: $B=0$ surface plot (i.e., variation of $\mu_{c l}$ with $\sigma_{1}$ and $\sigma_{2}$ for $\beta=0.4$ and $\mu_{i 2}=0.8$, where $\mu_{c l}$ isthe critical value of $\mu_{i 1}$ ) above which $\phi_{m}>0$ and below which $\phi_{m}<0$.

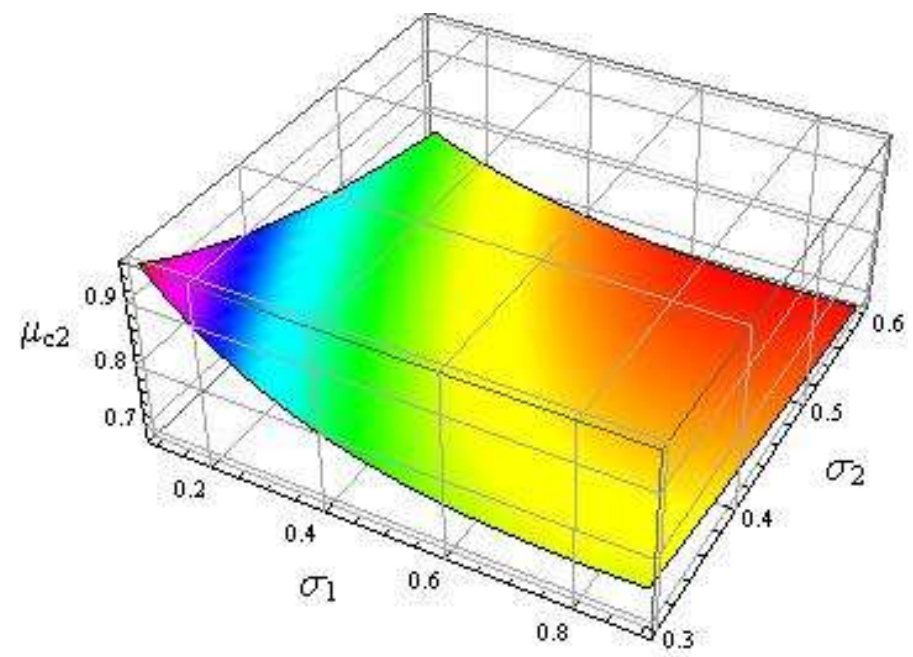

Figure 2: $B=0$ surface plot (i.e., variation of $\mu_{c 2}$ with $\sigma_{1}$ and $\sigma_{2}$ for $\beta=0.5$ and $\mu_{i 1}=0.4$, where $\mu_{c 2}$ is the critical value of $\mu_{i 2}$ ) above which $\phi_{m}>0$ and below which $\phi_{m}<0$.

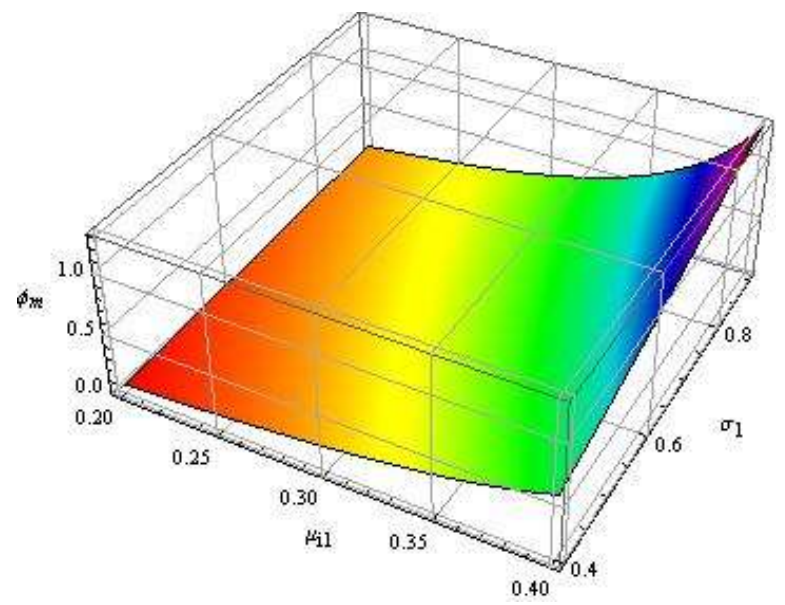


Figure 3: Variation of the amplitudes of solitary profiles for positive potential $\phi_{m}$ with $\mu_{i l}$ and $\sigma_{1}$ for $u_{0}=0.1$, $\mu_{i 2}=0.4, \sigma_{2}=0.41, \delta=20^{\circ}$ and $\beta=0.3$.

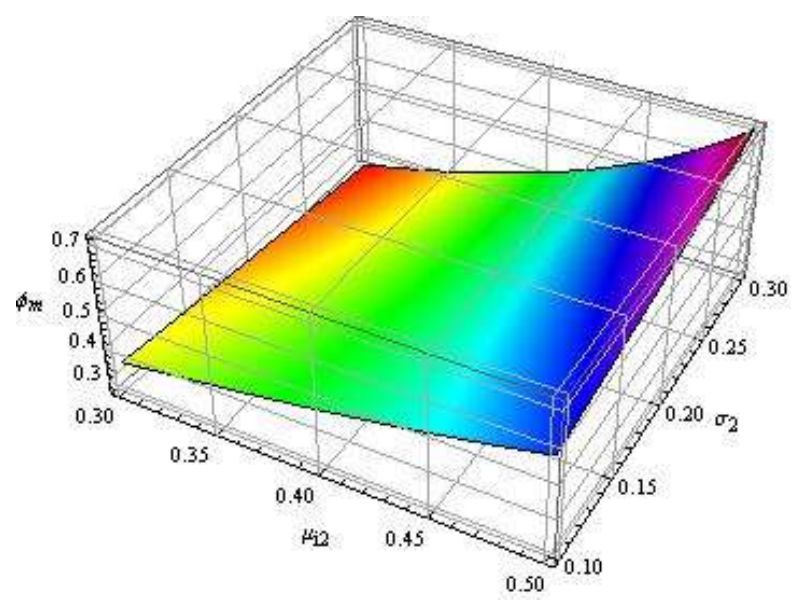

Figure 4: Variation of the amplitudes of solitary profiles for positive potential $\phi_{m}$ with $\mu_{i 2}$ and $\sigma_{2}$ for $u_{0}=0.1$, $\mu_{i l}=0.58, \sigma_{l}=0.2, \delta=20^{\circ}$ and $\beta=0.5$.

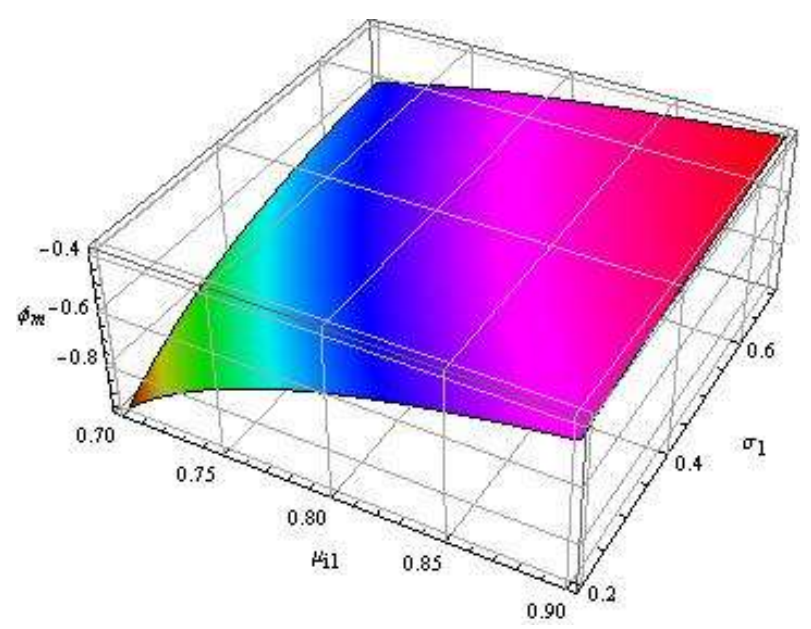

Figure 5: Variation of the amplitudes of solitary profiles for negative potential $\phi_{m}$ with $\mu_{i 1}$ and $\sigma_{1}$ for $u_{0}=0.1$, $\mu_{i 2}=0.4, \sigma_{2}=0.3, \delta=20^{\circ}$ and $\beta=0.3$.

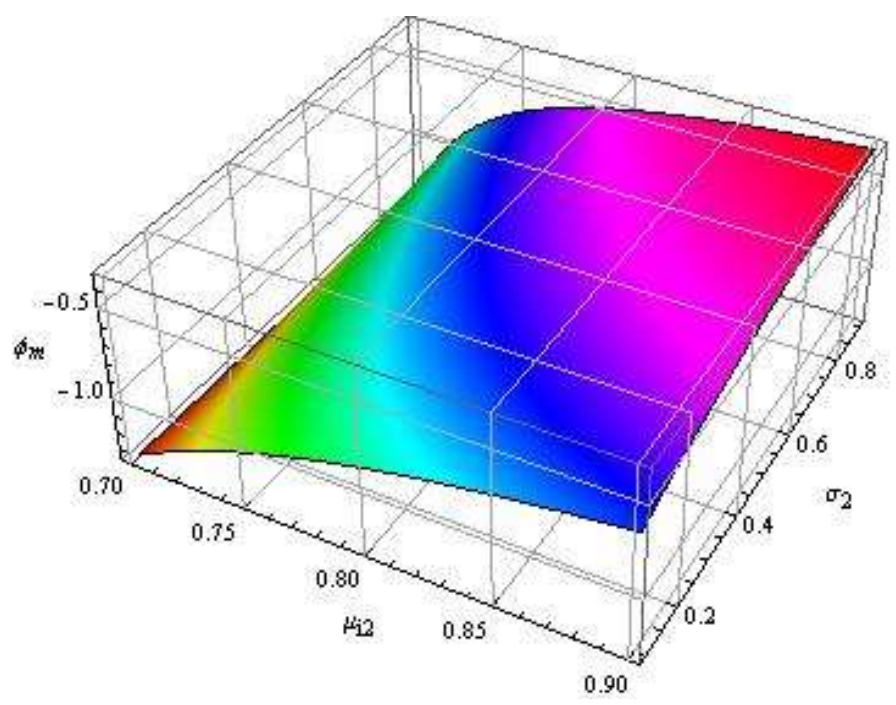

Figure 6: Variation of the amplitudes of solitary profiles for negative potential $\phi_{m}$ with $\mu_{i 2}$ and $\sigma_{2}$ for $u_{0}=0.1$, $\mu_{i 1}=0.3, \sigma_{1}=0.5, \delta=20^{\circ}$ and $\beta=0.3$. 


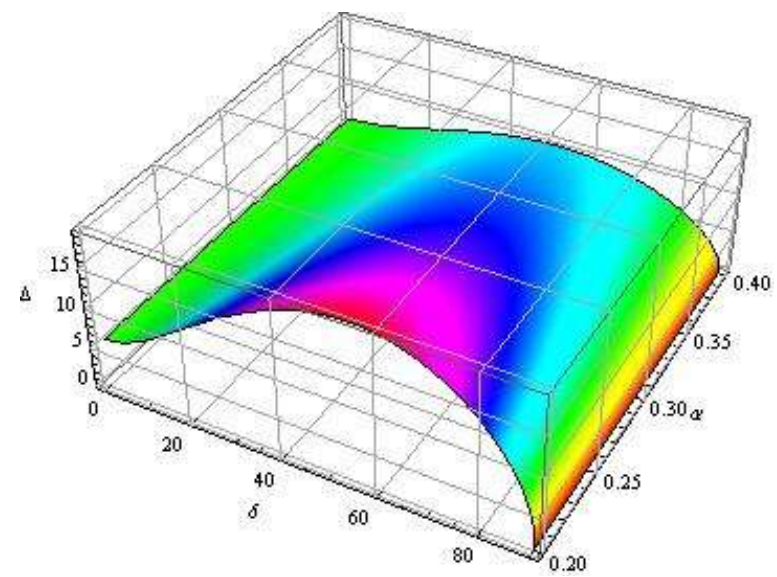

Figure 7: Variation of the width ( $\Delta$ ) of solitary waves with $\delta$ and $\alpha$ for $u_{0}=0.1, \mu_{i 1}=0.58, \mu_{i 2}=0.8, \sigma_{1}=0.2, \sigma_{2}=0.41$, and $\beta=0.3$.

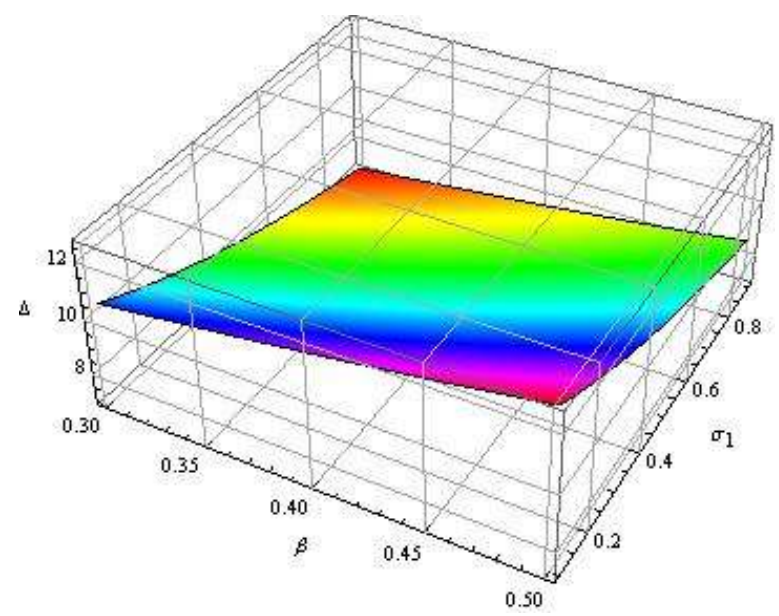

Figure 8: Variation of the width ( 4 ) of solitary waves with $\beta$ and $\sigma_{1}$ for $u_{0}=0.1, \mu_{i 1}=0.58, \mu_{i 2}=0.8, \sigma_{2}=0.41$, $\alpha=0.25$, and $\delta=20^{\circ}$.

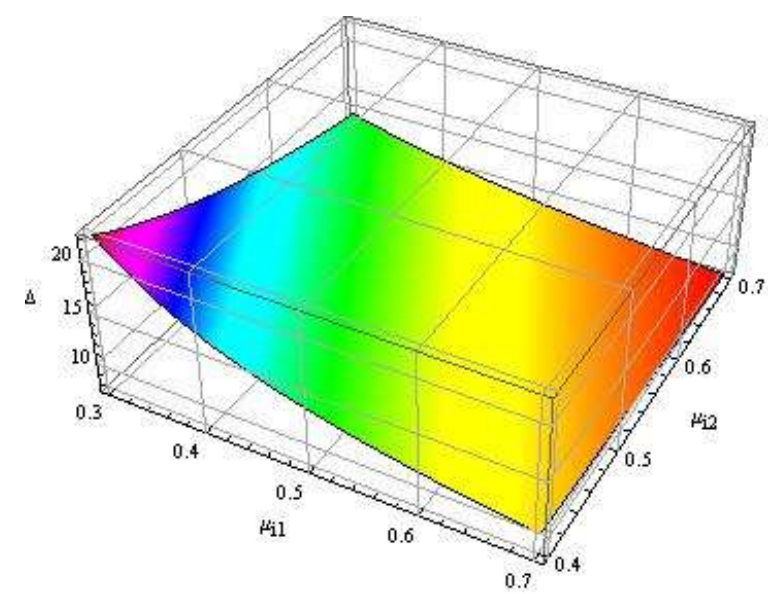

Figure 9: Variation of the width $(\Delta)$ of solitary waves with $\mu_{i 1}$ and $\mu_{i 2}$ for $u_{0}=0.1, \beta=0.3, \sigma_{1}=0.4, \sigma_{2}=0.41, \alpha=0.3$, and $\delta=20^{\circ}$.

\section{Numerical Analysis}

Figures 1 and 2 show $B=0$ surface plot showing the variation $\mu_{c l}$ and $\mu_{c 2}$, respectively, with $\sigma_{1}$ and $\sigma_{2}$, where $\mu_{c 1}\left(\mu_{c 2}\right)$ is the critical value of $\mu_{i l}\left(\mu_{i 2}\right)$. The figures indicate that the upper (lower) region of the surface corresponds to $B>0(B<0)$, i.e., corresponds to the positive (negative) DASWs. The figures also indicate that the critical values of $\mu_{i 1}$ and $\mu_{i 2}$ decrease, as the values of both $\sigma_{1}$ and $\sigma_{2}$ increase.It is observed from eqns. (15), (22), 
(23), (27), and $\phi_{m}=3 u_{0} / \delta_{1}$ that the amplitude $\left(\phi_{m}\right)$ is the nonlinear function of $\mu_{i 1}, \mu_{i 2}, \sigma_{1}, \sigma_{2}, \delta$, and $\beta$. The variation of $\phi_{m}$, for positive and negative potential, with $\mu_{i 1}, \mu_{i 2}, \sigma_{1}$, and $\sigma_{2}$ are shown in figs. 3-6. Fig. 3 shows the variation of the amplitudes of the positive $\operatorname{SWs}\left(\phi_{m}>0\right)$ with $\mu_{i 1}$ and $\sigma_{1}$ for $u_{0}=0.1, \mu_{i 2}=0.4, \sigma_{2}=0.41, \delta=20^{\circ}$ and $\beta=0.3$. This shows that the magnitude increases with increasing both values of $\mu_{i 1}$ and $\sigma_{1}$. Fig. 4 shows the variation of the amplitudes of the positive SWs $\left(\phi_{m}>0\right)$ with $\mu_{i 2}$ and $\sigma_{2}$ for $u_{0}=0.1, \mu_{i 1}=0.58, \sigma_{1}=0.2, \delta=20^{\circ}$ and $\beta=0.5$. This figure shows that the amplitude decreases (increases) with increasing the value of $\sigma_{2}$ for lower (higher) value of $\mu_{i 2}$, whereas the magnitude of the amplitude increases with increasing the value of $\mu_{i 2}$.Fig. 5 shows the variation of the amplitudes of the negative SWs $\left(\phi_{m}<0\right)$ with $\mu_{i 1}$ and $\sigma_{1}$ for $u_{0}=0.1, \mu_{i 2}=0.4, \sigma_{2}=0.3, \delta=20^{\circ}$ and $\beta=0.3$. The figure indicates that the amplitude of the negative solitary potential decreases with increasing the values of both $\mu_{i 1}$ and $\sigma_{1}$. Fig. 6 shows the variation of the amplitudes of the negative $\operatorname{SWs}\left(\phi_{m}<0\right)$ with $\mu_{i 2}$ and $\sigma_{2}$ for $u_{0}=0.1$, $\mu_{i l}=0.3, \sigma_{I}=0.5, \delta=20^{\circ}$ and $\beta=0.3$. This shows that the amplitude of the negative solitary potential deceases with increasing the values of both $\mu_{i l}$ and $\sigma_{l}$. This is to be noted that the amplitude of the external magnetic field has the significant effect only on the width, but not on the amplitude of these solitary waves.

It is found from eqns. (15), (22), (24), (27), and $\kappa=\sqrt{u_{0} / 4 \delta_{2}}$ that the width $(\Delta=1 / \kappa)$ is a nonlinear function of $\delta, \alpha, \mu_{i 1}, \mu_{i 2}, \sigma_{1}, \sigma_{2}$, and $\beta$. The variations of the width, for positive and negative SWs, with $\delta, \alpha, \mu_{i l}$, $\mu_{i 2}, \sigma_{1}$, and $\beta$ are represented in figs. 7-9. Fig. 7 shows how the width ( $\Delta$ ) of solitary waves varies with $\delta$ and $\alpha$ for $u_{0}=0.1, \mu_{i 1}=0.58, \mu_{i 2}=0.8, \sigma_{1}=0.2, \sigma_{2}=0.41$, and $\beta=0.3$. This figure shows that the width increases with $\delta$ for the lower range, i.e., from $0^{\circ}$ to about $55^{\circ}$, but decreases with $\delta$ for higher range, i.e., from about $55^{\circ}$ to $90^{\circ}$, and as $\delta \rightarrow 0^{\circ}$, the width goes to zero. This also shows that the width decreases with increasing $\alpha$, and is valid only for $\delta<90^{\circ}$. Fig. 8 shows how the width ( $\triangle$ ) varies with $\beta$ and $\sigma_{1}$ for $u_{0}=0.1, \mu_{i 1}=0.58, \mu_{i 2}=0.8, \sigma_{2}=0.41, \alpha=0.25$, and $\delta=20^{\circ}$. This figure indicates that the width of the SWs increases (decreases) with increasing the value of $\beta\left(\sigma_{1}\right)$. Fig. 9 shows the variation of the width $(\Delta)$ of solitary waves with $\mu_{i 1}$ and $\mu_{i 2}$ for $u_{0}=0.1, \beta=0.3, \sigma_{1}=0.4, \sigma_{2}=0.41$, $\alpha=0.3$, and $\delta=20^{\circ}$. The figure shows that the width decreases with increasing the both values of $\mu_{i 1}$ and $\mu_{i 2}$. We have not shown the change of width with $\sigma_{2}$ physically, but observed that the width of the SWs decreases with increasing $\sigma_{2}$, when other parameters remain unchanged.

\section{Discussion}

We have considered magnetized dusty plasma system consisting of negatively charged dust fluid, Boltzmann distributed electrons, and nonthermal ions of distinct temperatures, and have studied DASWs associated with both positive and negative potentials by deriving the $\mathrm{ZK}$ equation. We have found that, depending on the values of $\mu_{i 1}, \mu_{i 2}, \sigma_{1}, \sigma_{2}, \delta$, and $\beta$, the SWs may become associated with either positive or negative potential. We have seen that, as the values of $\mu_{i 1}, \mu_{i 2}, \sigma_{1}$, and $\sigma_{2}$ increase, the amplitude of the positive (negative) SWs increases (decreases), and their width increases (decreases) with the increase of the $\beta$ value $\left(\mu_{i l}\right.$, $\mu_{i 2}, \sigma_{1}$, and $\sigma_{2}$ values). We have found that as the value of $\delta$ increases, the width of the SWs (for both positive and negative potentials) increases for the lower range of $\delta$ (from $0^{\circ}$ to about $55^{\circ}$ ) but decreases for its higher range (from about $55^{\circ}$ to $90^{\circ}$ ). As $\delta \rightarrow 90^{\circ}$, the width goes to zero, and the amplitude goes to $\infty$. It is likely that, for large angles, the assumption that the waves are electrostatic is no longer valid, and we should look for fully electromagnetic structures. It is found that the magnitude of the external magnetic field $\mathbf{B}_{0}$ has no direct effect on the SW amplitude. However, it does have a direct effect on the width of the SWs, and have found that, as the magnitude of $\mathbf{B}_{0}$ increases, the width of the wave decreases, i.e., the magnetic field makes the solitary structures spikier.Therefore, it is found that the effect of nonthermal ions of distinct temperature and external magnetic field significantly modify the basic properties of the DASWs.

The ranges $\left(\beta=0.1-0.9, \alpha=0.1-0.9, \mu_{i 1}=0.1-0.9, \mu_{i 2}=0.1-0.9, \sigma_{1}=0.1-0.9, \sigma_{1}=0.1-0.9\right)$ of the dusty plasma parameters used in this numerical analysis are very wide. Therefore, the dusty plasma parameters corresponding to space and laboratory dusty plasmas are certainly within these ranges. So, our present results may be useful for understanding the localized electrostatic disturbances in space and laboratory dusty plasmas.

\section{References}

[1]. P. K. Shukla and A. A. Mamun, Introduction to Dusty Plasma Physics (IOP Publishing Ltd., Bristol, 2002).

[2]. F. Verheest, Waves in Dusty Plasmas (Kluwer, Dordrecht, 2000).

[3]. C. K. Goertz, Rev. Geophys. 27, 271 (1989).

[4]. M. Horányi, Annu. Rev. Astron. Astrophys. 34, 383 (1996).

[5]. A. A. Mamun and S. Islam, J. Geophys. Res. 116, A12323 (2011)

[6]. M. M. Masud, M. Asaduzzaman, and A. A. Mamun, Phys. Plasmas 19, 103706 (2012).

[7]. N. N. Rao, P. K. Shukla, and M. Y. Yu, Planet Space Sci. 38, 543 (1990).

[8]. A. Barkan, R. L. Merlino, and N. D'Angelo, Phys. Plasmas 2, 3563 (1995).

[9]. A. A. Mamun and P. K. Shukla, Geophys. Res. Lett. 29, 1870 (2002).

[10]. A. A. Mamun and P. K. Shukla, Geophys. Res. Lett. 31, L06808 (2004).

[11]. F. Melandsø, Phys. Plasmas 3, 3890 (1996).

[12]. M. Rosenberg and D. A. Mendis, IEEE Trans. Plasma Sci. 23, 177 (1995)

[13]. F. Verheest and S. R. Pillay, Nonlin. Processes Geophys. 15, 551 (2008).

[14]. H. Alinejad, Astrophys. Space Sci. 337, 223 (2012). 
[15]. N. R. Kundu and A. A. Mamun, J. Plasma Phys. 78, 677 (2012).

[16]. H. Matsumoto et al., Geophys. Res. Lett. 21, 2915 (1994).

[17]. W. C. Feldman et al., J. Geophys. Res. 88, 96 (1983).

[18]. R. Lundlinet al., Nature 341, 609 (1989).

[19]. R. Bostr"omet al., Phys. Rev. Lett. 61, 82 (1988).

[20]. M. Temerinet al., Phys. Rev. Lett. 48, 1175 (1982).

[21]. P. O. Dovneret al., Geophys. Res. Lett. 21, 1827 (1994).

[22]. Y. Futaanaet al., J. Geophys. Res. 151, (2003).

[23]. R. A. Cairns et al., Geophys. Res. Lett. 22, 2709 (1995).

[24]. A. A. Mamun, R. A. Cairns, and P. K. Shukla, Phys. Plasmas 3, 2610 (1996).

[25]. M. M. Lin and W. S. Duan, Chaos, Solitons\&Fractals 33, 1189 (2007).

[26]. I. Tasnimet al., Chaos 23, 013147 (2013).

[27]. H. Asgari, S. V. Muniandy, and C. S. Wong, Phys. Plasmas 20, 023705 (2013).

[28]. T. S. Gill, H. Kaur and N. S. Saini, J. Plasma Phys. 70, 481 (2004).

[29]. A. A. Mamun, Astrophys. Space Sci. 260, 507 (1999).

[30]. I. Tasnim, M. M. Masud, and A. A. Mamun, Astrophys. Space Sci. 343,647 (2013).

[31]. M. Asaduzzaman and A. A. Mamun, J. Plasma Phys. 78, 601 (2012).

[32]. M. M. Masud and A. A. Mamun, JETP Lett. 96, 765 (2013).

[33]. N. Y. Tanisha et al., Astrophys. Space Sci. 353, 137 (2014).

[34]. M. M. Masud and A. A. Mamun, J. Plasma Phys. 1, 1 (2013).

[35]. H. Derfler, and T. C. Simonen, Phys. Fluids 12, 269 (1969).

[36]. A. A. Mamun and P. K. Shukla, J. Geophys. Res. 107, 1135 (2002).

[37]. S. T. Shuchy, A. Mannan, and A. A. Mamun, IEEE Trans. Plasma Sci. 40, 1 (2012).

[38]. M. S. Alam, M. M. Masud, and A. A. Mamun,Braz. J. Phys 45, 95 (2015)

[39]. N. Duboulozet al.,Geophys. Res. Lett. 18, 155 (1991).

[40]. M. Berthomieret al., Phys. Plasmas 7, 2987 (2000).

[41]. I. Tasnim, M. M. Masud, and A. A. Mamun, Astrophys. Space Sci. 343, 647 (2013).

[42]. D. Dorranian and A. Sabetkar, Phys. Plasmas 19, 013702 (2012).

[43]. N. R. Kunduet al., Astrophys. Space Sci. 343, 279 (2013).

[44]. H. Washimi, T. Taniuti, Phys. Rev. Lett. 17, 996 (1966). 